\title{
On the viability of the No Alternatives Argument
}

\author{
Tushar Menon* \\ Balliol College, University of Oxford
}

August 25, 2018

\begin{abstract}
If we cannot directly empirically test the claims of particular scientific theory, then it would be nice to have some other criteria with which to assess its viability. In his 2013 book, String Theory and the Scientific Method, Richard Dawid aims to develop such criteria, with an eye to vindicating research programs in disciplines where direct empirical data is scant or non-existent. In an accompanying paper, Dawid, Hartmann and Sprenger formalise Dawid's so-called 'No Alternatives Argument' (NAA) using a generalised Bayesian framework, as a first step towards formalising Dawid's entire research programme (which itself relies on two further arguments). In this paper, I argue that the formalisation of the NAA cannot play the central role in Dawid's programme as intended. This is based on the observation that not all confirmation is non-negligible confirmation. For Dawid's programme to be useful, it must demonstrate the viability not just of non-empirical theory confirmation, but of non-negligible non-empirical theory confirmation. I argue that Dawid et al.'s appeal to Bayesian confirmation theory to formalise his NAA cannot guarantee non-negligible confirmation. As a result, I conclude that if Dawid's overall project is to succeed, it must do so without the NAA formalised in this way.
\end{abstract}

*email: tushar.menon@balliol.ox.ac.uk 


\section{Contents}

1 Introduction $\quad 2$

2 The Dawid Programme 5

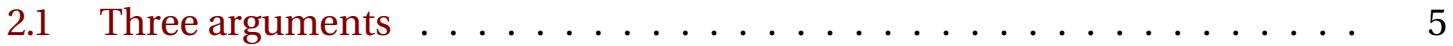

2.2 Non-empirical Bayesian confirmation . . . . . . . . . . . 7

2.2.1 The formal setup $\ldots \ldots \ldots \ldots$

2.2 .2 Non-negligibility . . . . . . . . . . . . . . . 9

3 The objection 10

3.1 Significance . . . . . . . . . . . . . . . . . 10

3.2 The Gerrymandering objection . . . . . . . . . . . . . . . . . 14

4 Conclusion $\quad 19$

\section{Introduction}

Depending on whom one asks, String Theory is anything between the biggest scandal in theoretical physics and its greatest modern achievement. Proponents of the latter view argue that String Theory is the ultimate unified theory, which potentially provides a complete description of all phenomena. Opponents argue that, insofar as String Theory makes no real, testable predictions, any claims to it being the final theory are premature at best.

This reference to testable predictions brings to focus an important question for the philosopher of science: how do we choose between rival hypotheses which purport to describe, predict and explain the phenomena we observe? A popular move involves assigning so-called 'evidence-based' prior probabilities to competing hypotheses and subjecting them to a Bayesian confirmation algorithm. This does not limit the number of hypotheses that could account for a particular data set, rather it provides, in theory at least, a quantitative way of assessing how well a given hypothesis responds to extant empirical data, or data from tests of novel experimental predictions made by the theory. After all, in practice, we never really find ourselves in a situation where we have more than two or three viable alternative hypotheses, and even then, it is rare that they all account for all observed data.

What if there are no novel experimental tests or extant empirical data available for a particular theory? In a series of publications [1, 2, 3, 4, 5, 7], Richard Dawid proposes that we respond to the problem of confirmation in this context in a different way: rather than assigning evidence-based priors, Dawid suggests we use an alternative set of inputs, which he calls 'Limitation to Scientific Underdetermination' inputs. So far, so good: insofar as it is a piece of mathematics, Bayes' Theorem is indifferent to the source of its inputs. 
These novel inputs are an important aspect of Dawid's more general attempt to develop an account of confirmation which is tied to limitation to scientific underdetermination. On such an account, empirical data is useful insofar as it constrains theory space-by limiting the number of alternative theories consistent with a given set of data points. But, Dawid proposes, there are other important ways to limit scientific underdeterminationin particular, he suggests that we can use the observation that scientists have not found adequate alternatives to a paticular theory, together with some further pretheoretic requirements as a stand-in for empirical data. Dawid's programme, described in more detail in $\$ 2$, relies on three mutually-reinforcing arguments, the 'No Alternatives Argument' (NAA), the 'Unexpected Explanatory Coherence Argument' (UEA) and the 'Meta-Inductive Argument' (MIA), to provide confirmation to scientific theories like string theory. As a step towards formalising this research programme, Dawid, Hartmann and Sprenger introduced a formalisation of the NAA [7]. In this paper, I aim to demonstrate that the NAA, on its own cannot generate non-negligible confirmation, so even though it forms an indispensable part of Dawid's programme of confirmation, it cannot feature in a programme of nonnegligible confirmation. This paper is not intended as a argument against the viability of the entire research programme; rather it should be taken to question the centrality of the NAA to a programme of non-negligible non-empirical confirmation.

The NAA is suceptible to what I will refer to as the gerrymandering objection-that confirmation cannot be guaranteed to be significant without an artificially chosen set of priors. ${ }^{1}$ The requirement of significance of confirmation is exemplified by argument from a familiar problem: does observing a non-black non-raven confirm the hypothesis that all ravens are black? [9] Bayesian Confirmation Theory argues, perhaps counter-intuitively, that it does, but the degree of confirmation in question is so small as to be negligible. Not all confirmation, then, is non-negligible confirmation. ${ }^{2}$ For Dawid's programme to be successful, it must demonstrate not only the viability of non-empirical theory confirmation, but of non-negligible ${ }^{3}$ non-empirical theory confirmation. If Dawid cannot do this, (and the argument in this paper against the ability of the NAA to guarantee significant confirmation gives us reason to doubt the overall viability of his programme) then the project would present us with nothing more than a mere formal curiosity. It would be a mathematical residue of the fairly uncontroversial intuition that there are certain theoretical virtues that a theory ought to have (consistency, for example ${ }^{4}$ ). Whatever they may be, it seems wrong to suggest that a theory can be significantly confirmed merely in virtue of displaying these features.

\footnotetext{
${ }^{1}$ Logically, this is equivalent to the disjunction of the claims (i) that significant confirmation is impossible and (ii) significant confirmation is achieved by stipulating a contrived set of priors. This disjunction is reflected in the structure of $\$ 3$.

${ }^{2}$ The role played by the non-negligibility requirement is discussed in more detail at the end of $\$ 3.1$.

${ }^{3}$ In this paper, I take 'non-negligible confirmation' to be synonymous with 'significant confirmation'.

${ }^{4}$ Although even this is not uncontrovesial; Vickers [12] argues against the notion that consistency is an important super-empirical virtue.
} 
The only way in which the NAA stands a chance of evading the problem of negligible confirmation is by associating some highly contrived probability density functions to the priors. Specifying these functions to the level of detail required to establish non-negligible confirmation makes the project run afoul of the gerrymandering objection, discussed in detail in $\$ 3.2$.

Dawid's programme is intended to be neutral on the theories it tests. It is, however, useful to have a concrete example in mind of a theory that would benefit from such a programme. Here, I choose to use Dawid's primary example, String Theory. The less physics-inclined reader is free to substitute any other theory with scant direct experimental evidence (Dawid, for example, also discusses paleontology in his book). It is important to distinguish three different claims about string theory that have the potential to get mixed together. The first claim is immodest: it says that String Theory is the final theory of everything. The second claim is more modest: it says that String Theory is likely to be a viable theory of all known interactions up to some high energy scale. The third is that String Theory is worth doing. Whilst a reading of Dawid's book as making the first claim may well be a viable one ${ }^{5}$ in this paper, I interpret the book as a defence of the second claim, that String Theory is viable theory of all known interaction (in virtue of which it is worth doing). I begin, in $\$ 2$ with a brief recapitulation of Dawid's programme and the mathematics of non-empirical theory confirmation. In $\$ 3$, I present and discuss two objections to the use of the formalised NAA. None of this is intended as an argument either that research in String Theory is not justified or that Dawid's programme is untenable across the board; I merely contend that String Theory research cannot be justified to a non-negligible degree purely by appeal to a programme centered on the NAA as formalised by Dawid et al. ${ }^{6}$

\footnotetext{
5 Two observations make it quite plausible that Dawid is making the stronger claim. Firstly, that half the book is dedicated to discussions about String Theory as being the final theory, and the impact that it has on the debate over scientific realism, I think it is not implausible to read Dawid as making the stronger claim. Secondly, the following quote from his most recent paper, [6, pp.4-5]: 'One might...consider a more restrained and therefore possibly less contestable point of view. On that view the crucial question for the working scientist is simply whether or not it makes sense to work on a given theory... I do not concur that what is at stake in non-empirical theory assessment can be reduced to the pragmatic issue of deciding upon research strategies... [I]n the end, a strong commitment to working on a particular theory hinges on the question whether there exists a good reason for having trust in the theory's viability.

${ }^{6}$ Although the target of the analysis in this paper is Dawid et. al's formalisation of the NAA, and Dawid's accompanying inferences about String Theory confirmation, I should mention that the arguments provided here also serve as a rebuttal to Eva and Hartmann's [8] claims that the absence of reasons for a belief can be seen as reasons against that belief. This is on the grounds that the analysis provided by Eva and Hartmann are, in all relevant ways, isomorphic to those provided by Dawid et. al [7]. To flesh this claim out in more detail, however, would be tangential to the thrust of this paper-I have done so in a companion paper [?]. I am grateful to an anonymous referee for drawing my attention to Eva and Hartmann's paper.
} 


\section{The Dawid Programme}

\subsection{Three arguments}

Dawid addresses a range of interrelated issues in the philosophy of String Theory, and the philosophy of science more generally, in String Theory and the Scientific Method. His programme is centred on using non-empirical criteria to limit the number of viable empirically adequate theories. To mount a criticism, or even engage in a discussion of all the important philosophical issues raised by the book would be well beyond the scope of a short paper like this. I confine myself, therefore, to the core of Dawid's programme-the NAA as formalised using (non-empirical) Bayesian confirmation theory. In this section, I present a summary of Dawid's programme, based on his book [3], as well as companion papers $[1,2,4,5,7]$.

\section{The No Alternatives Argument}

Dawid makes the following claim about the three arguments:

...each of the individual strategies of non-empirical confirmation in isolation does constitute confirmation but cannot be established to be significant. As we will see, significant confirmation can only be made plausible based on two or even three arguments of non-empirical confirmation in conjunction. [6]

The centrepiece of Dawid's programme is the No Alternatives Argument (NAA) - the other two arguments are intended to shore up our credence in the claim that String Theory is viable. However, absent a guarantee of significant confirmation from formalisations of the other two arguments (which, as of the time of writing, are indeed absent), we need a guarantee that the confirmation provided by the NAA is non-negligible. The NAA forms the basis of the Bayesian analysis that Dawid uses to claim that String Theory is nonempirically confirmed. The mathematical details of the construction are presented in $\$ 2.2$, where they are scrutinised. In this section, I provide a formal overview of the important moving parts of the NAA:

P1: Scientists have not yet found an alternative hypothesis to $H$, which satisfies certain constraints, ${ }^{7}$ despite considerable effort having been spent in trying to do so.

P2: If scientists have not yet found an alternative to $H$, then either (i) it is likely that there are few or no alternatives to $H$ or (ii) it is too difficult for scientists to find alternatives to $H$.

\footnotetext{
${ }^{7}$ Dawid's a priori constraints on theories fall into three categories—-they must be able to account for extant empirical data $(\mathcal{D})$, they must provide distinguishable predictions for the outcomes of future experiments (call this set $\mathcal{E}$ ), and they must satisfy a set of scientificality constraints $C$. ' $C$ specifies what counts as a scientific theory. Only those theories that meet the scientificality conditions count as possible theories. Scientificality conditions are themselves volatile to a given degree and may change in time' [5, p. 8]. These constraints are discussed in detail in $[3,5]$. In this paper, I assume that these constraints are unproblematically enforcible.
} 
P3: It is not too difficult for scientists to find alternatives to $H$.

P4: If scientists have not yet found an alternative to $H$, then it is likely that there are few or no alternatives to $H$.

P5: If it is likely that there are few or no alternatives to $H$, then scientists should have a high degree of trust in $H^{8}$

From this, one concludes:

Conclusion: Scientists should have a high degree of trust in $H .{ }^{9}$

To formalise this argument, Dawid takes the conclusion to be equivalent to the statement that the proposition $T$ is confirmed if $P(T \mid F)>P(T)$, where $T$ is the proposition that $H$ is empirically adequate and $F$ is the proposition that states the observation that, despite considerable effort having been spent in trying to do so, scientists have not yet found an alternative a hypothesis to $H$ which satisfies certain constraints. In $\$ 3.1$, I argue that this jump is not warranted; a non-negligibility condition needs to be met before the two statements can be taken to be equivalent.

$\mathbf{P 1}$ is taken to be an empirical fact; for the purpose of this paper, I treat it as such. ${ }^{10}$ Dawid et al.'s analysis in [7] demonstrates that $P(T \mid F)>P(T)$ confirms the inclusive disjunction of two propositions: (i) there are few or no alternatives to $H$ and (ii) the problem of finding alternative theories is too difficult for scientists given cognitive, technological and other methodological and physical constraints. In order to tip the balance in favour of confirmation of the first disjunct, Dawid relies on two further arguments, the metainductive argument (MIA) and the unexpected explanatory coherence argument (UEA). The result of these arguments is summarised in P3. Granting, for the sake of argument, the truth of P3, the disjunction in $\mathbf{P 2}$ reduces to the proposition P4. The likelihood of truth of $\mathbf{P 4}$ and $\mathbf{P 5}$ are undermined by the gerrymandering objection of §3.2. Before turning to the objections, it is worth presenting two further arguments that Dawid uses to bolster the NAA, the unexpected explanatory coherence argument and the meta-inductive argument.

\section{The Unexpected Explanatory Coherence Argument}

For such theories, the combination of the NAA and the MIA still does not allow us to conclude that theory space has been constrained significantly, thus confirming $H$. Here,

\footnotetext{
${ }^{8}$ This proceeds via a sub-premise, that the smaller the number of alternatives to a hypothesis, the higher the probability that the hypothesis is true. van Basshuysen [10] correctly points out that this premise is not as innocent as it sounds.

${ }^{9}$ The NAA as presented by Dawid does not include reference to a 'high degree of trust.' However, since the basis of the argument in this paper is that the NAA (and, indeed all of non-empirical confirmation) is only useful if it gives us sufficiently good reason to believe in the viability of $H$, I include it as a part of the conclusion of the NAA.

${ }^{10}$ A more accurate description would perhaps be that $\mathbf{P 1}$ is a meta-empirical piece of data, since it is not (directly or straightforwardly) predicted by the theory in question.
} 
Dawid introduces a third and final argument, the Unexpected Explanatory Coherence Argument. According to this argument, if $H$ was developed with a view to accounting for one particular type of phenomenon, but unexpectedly accounts for other phenomena outside the intended domain of the hypothesis, then this makes it more likely that the observation that $P(T \mid F)>P(T)$ is the result of there genuinely being few or no alternatives to $H$, rather than that it was too difficult for scientists to find alternatives. Structurally, it shores up the NAA in the same way as the MIA does. Dawid claims that this is the 'non-empirical "cousin" of novel confirmation' [5, p. 15].

\section{The Meta-Inductive Argument}

It is quite difficult to invent predictively successful theories. Much easier is to invent theories which can account for extant data. However, the history of science shows multiple instances of scientists coming up with research programmes which have predictively successful theories. If $H$ is the result of research in the context of a programme that has, in the past, had much predictive empirical success, then this serves as an inductive argument in favour of other theories in the research programme. This is an induction, therefore, at the meta-level. This makes the lack of alternatives more likely an explanation of the confirmation than the suggestion that finding alternatives is too difficult for scientists.

Dawid uses the MIA (combined with the Bayesian analysis on the NAA) to argue for the explanation that theory space is genuinely limited to no or few alternatives to String Theory, given that it is part of the same research programme that gave us the now highly empirically confirmed Standard Model. But even if we were to grant this, there is still the question of how one ought to use Dawid's method for hypotheses which do not clearly sit in the same research programme as previously predictive successful theories.

\subsection{Non-empirical Bayesian confirmation}

Bayesian confirmation of scientific hypotheses proceeds iteratively as relevant empirical evidence is accumulated. Assume, in order to incorporate different positions on the scientific realism debate, that all we wish to confirm is the empirical adequacy ${ }^{11,12}$ of a scientific hypothesis. Bayes' Theorem follows from the definition of conditional probabilities and, in its simplest form, reads:

$$
P(T \mid E)=\frac{P(E \mid T)}{P(E)} \cdot P(T)
$$

When applied to scientific hypotheses, the variables in the above formula can usefully

\footnotetext{
${ }^{11}$ I refer to one canonical presentation of empirical adequacy from van Fraassen, 'a theory is empirically adequate exactly if what it says about the observable things, and events in the world, is true-exactly if it 'saves the phenomena' [11, p. 12].

${ }^{12}$ More recently, Dawid uses the term 'viability' in place of empirical adequacy. By 'viability', he means, 'the agreement of the theory's predictions will all empirical data that can possibly be collected within a given regime.' [6, p. 9]
} 
be given the following interpretation. $T$ is the proposition that $H$ is empirically adequate. $E$ is a proposition expressing some piece of empirical evidence. Given some prior assignment of a probability of truth of $T$, Bayesian confirmation theory says that, in light of some evidence $E$, the probability that the $T$ is true is given by the formula above. To count as confirmation, ${ }^{13}$ it simply needs to be the case that $P(T \mid E)>P(T)$. One assumes that the evidence represented in $E$ is relevant to the hypothesis $H$ (to use a line from Dawid, '[that] $E$ falls into the (broadly construed) intended domain of $H$ [and] $E$ is logically or probabilistically related to $H^{\prime}$ [7, p. 215]).

In the absence of empirical data, it seems like the Bayesian project is doomed, given the apparent irrelevance of non-empirical data to a hypothesis. But Dawid argues that the following two-step procedure might render Bayesian Confirmation Theory appropriate to the task. Consider the observation that no alternatives to $H$ have been discovered despite a great deal of effort on the part of the scientific community. Call this proposition $F$. Although $F$ is not relevant to the hypothesis $H$, if one can find a proposition that is relevant, in some significant sense, to $H$ and, at the same time, predicts the content of $F$, then it might serve as a surrogate for empirical data. Dawid proposes that the set of propositions, $Y_{k}$ of the form 'There are $k$ alternatives to hypothesis $H$ ' fits the bill. It is arguably highly plausible that that set of propositions, for some small value of $k$, increases the probability of $F$ being true. That it adequately 'mediates the connection' between $T$ and $F$ is less obvious.

\subsubsection{The formal setup}

It is useful to bear in mind the aim of the analysis that follows: to show that we can legitimately conclude that $P(T \mid F)>P(T)$. To that end, we need some more machinery. We already have the propositions $Y_{k}$. To this we add an auxiliary set of variables $D_{j}$ where $j$ takes values in the natural numbers, representing the 'difficulty' associated with finding alternatives, that has to do with 'the cleverness of the scientists, or the available computational, experimental and mathematical resources' [7, p. 218]. While the value of $D_{j}$ does not directly affect the probability of $H$ being adequate, it does affect the conditional probability of $\left(F \mid Y_{k}\right)$ being true.

With this setup in place, the authors impose the following five constraints on functions defined on these variables: ${ }^{14}$

\footnotetext{
${ }^{13}$ This idiosyncratic use of word 'confirmation' is liable to lead to confusion about what is being claimed by Dawid. 'Confirmation', as used in ordinary conversation, is often taken to mean something like 'establishment of the truth or correctness of', while in the Bayesian (and Dawidian) sense, is closer to something like 'endorse' or 'give credence to', and merely refers to an increase in the probability of truth (or empirical adequaxcy, or whatever else is made to stand in for truth) of a hypothesis.

${ }^{14}$ More recently, Dawid has attempted to incorporate certain non-negligibilty constraints. These are conditions which, should they be fulfilled by a programme of non-empirical theory assessment, 'has a plausible path towards being significant.' Briefly, the three conditions are [6, p. 17]:

1. The observations... should be about the external world rather than merely about the system of scientists and their theories.

2. It should be possible to construe an argument of non-empirical confirmation based on 'soft' empiri-
} 
(i) that $T$ is independent of $F$ given $Y$. This captures the intuition that our belief in the empirical adequacy of $H$ is not altered by the knowledge that there are $k$ alternatives to $H$. (ii) $D$ is independent of $Y$. The number of alternatives does not depend on the factors which affect how difficult it is for scientists to find an alternative to $H$.

(iii) $f_{k j}:=P\left(F \mid Y_{k}, D_{j}\right)$ is non-increasing in $k$ for all $j$ and non-decreasing in $j$ for all $k$. This function encodes the constraint that a large number of alternatives increases (or keeps constant) the likelihood of finding alternatives to $H$. It also incorporates the constraint that increasing the difficulty of a problem decreases (or keeps constant) the likelihood of finding alternatives to $H$.

(iv) $t_{k}:=P\left(T \mid Y_{k}\right)$ is non-increasing in $k$. In other words, as the number of alternatives increases, the probability that the hypothesis $H$ is empirically adequate decreases (or stays the same).

(v) There is at least one pair of distinct natural numbers, $i, k$ such that $y_{i} y_{k}>0$, where $y_{k}=P\left(Y_{k}\right)$ and $f_{i j}>f_{k j}$ for some $j$ and $t_{i}>t_{k}$.

\subsubsection{Non-negligibility}

Making the values ascribed to priors more quantitatively precise is a standard problem in Bayesian Confirmation Theory, and not one whose resolution is within the scope of this paper. Luckily that problem does not need to be solved in order to undermine the power of the NAA. In what follows, I argue that the manner in which the values of the inputs are constrained by Dawid's programme makes them unsuitable to the task of providing non-negligible confirmation.

Insofar as Dawid's Programme requires that hypothesis $H$ be made more likely to be viable on confrontation with data regarding the lack of alternatives, his programme is successful. But, as alluded to in the introduction and footnote 2, it runs the risk of achieving this purely formal notion of confirmation (in the Bayesian sense) in a way that does not guarantee that scientists ought to have a significantly higher credence in the hypothesis $H$. Providing a precise mathematical boundary for what counts as nonnegligible is dependent on a number of factors specific to the problem in question. In the Ravens Paradox case, for example, it is not clear that there is, in fact, a precise lower bound for non-negligibility. But is is clear that, given the sheer number of events that qualify as tests of the hypothesis that all ravens are black (equivalently, all non-black things are non-ravens), the level of confirmation is tiny on any reasonable measure. I

cal confirmation of a meta-level hypothesis $Y$ by well specified non-empirical evidence $F$ which, in conjunction with a positive correlation between $Y$ and the viability of $H$, establishes that $F$ confirms $H$

3. Non-empirical confirmation should be applicable only to empirically predictive theories.

The idea behind incorporating these conditions is that they 'resemble empirical confirmation in crucial ways.' These merely refine the sorts of hypotheses subjected to his programme, rather than restructuring the entire programme. Insofar as the following criticism is applicable to any hypotheses subjected to Dawid's programme, these conditions are of no help to Dawid. 
contend that the level of confirmation that Dawid's programme provides is of the same order of magnitude, and to this, I ascribe the term 'negligible'. Providing an exact cut-off for negligibility would require arguments beyond the scope of this paper, but it should be pointed out that there are, indeed, certain values that one can ascribe to Dawid's credence functions that would arguably qualify as having led to significant confirmation. If, for example, it can be shown that there is a probability of approximately 1 that there are no alternatives to String Theory, then at least from the perspective of arguments in this paper, that qualifies as non-negligible confirmation. For the grey area in between, it is incumbent on Dawid to provide a justification for considering such confirmation significant.

Dawid, Hartmann and Sprenger [7] develop a Bayesian model for the NAA which they intend to form the foundations of a quantitative non-empirical theory confirmation programme. In \$3.1, I show how, under plausible assumptions, this model simply cannot account for non-negligible confirmation. In other words, even if the Bayesian updating step warrants an increase in priors for a given hypothesis $H$, given Dawid's analysis, the increase in these priors will not be guaranteed to be significant. On the surface, this resembles a more general argument against the Bayesian confirmation programme-that the Bayesian updating step requires for confirmation only relative, rather than absolute increase in priors, and that there is in general, no known way to make quantititavely precise the procedure of assigning those probabilities. But this criticism leaves open the option that such a procedure could exist and that we have just not found it as yet. My criticism is stronger-I argue that for Dawid's version of Bayesian Confimation, subject to some plausible restrictions on the behaviour of the relevant probability density functions, such a procedure either does not exist, or is susceptible to a circularity objection.

\section{The objection}

In this section, I detail the manner in which Dawid et. al's programme runs afoul of the gerrymandering objection by discussing five cases that jointly cover all the possible forms that the relevant probability functions might take. I begin, in $\$ 3.1$ by describing two cases in which significant confirmation is unobtainable. I conclude, in $\$ 3.2$ with three cases in which confirmation could be significant, but only at the cost of having highly gerrymandered priors or probability functions, i.e. falling prey to the gerrymandering objection.

\subsection{Significance}

Under all the assumptions presented in the last section, the authors prove that the $F$ can be treated as a substitute for $E$, and $P(T \mid F)>P(T)$. The details of the proof are in the appendix of [7]. The punch line is what matters. Having rephrased the Bayesian confirmation constraint as $P(T \mid F)-P(T)>0$, the authors deduce that this is equivalent to the following requirement, where $d_{j}$ is the probability that the problem of finding alternatives has a 
difficulty of $D_{j}$ :

$$
\sum_{i=1}^{\infty} \sum_{j=1}^{\infty} \sum_{i \neq k=1}^{\infty} d_{j} y_{i} y_{k}\left(t_{i}-t_{k}\right)\left(f_{i j}-f_{k j}\right)>0
$$

While the deduction, given the constraints, is unquestionably correct (and this is all the authors set out to prove), it does not capture the essence of a non-negligible confirmation of the hypothesis $H$. Let us split the sum into two components, by separating the sum over $d_{j}$. The remaining sum is over the product of three factors, $y_{i}, t_{i}$ and $f_{i j}$. One immediate problem with this setup (which is pointed out by Dawid et al. [7, pp. 225-226]) is that this product being greater than zero could be accounted for by a sufficiently high value of $d_{j}$. In other words, this analysis could just as legitimately be interpreted as showing that the problem of finding alternatives is just too difficult, for the reasons that are encoded in the definition of $D_{j}$. For Dawid et al., this is a problem that they address by appeal to the Meta-Inductive argument. They claim that the past successes of the research programme of particle physics makes it reasonable to assume that the problem of finding a theory of quantum gravity with the relevant constraints is not beyond the cognitive and practical resources of the best physicists. I am suspicious of this move and am inclined to believe that, whether or not there exists some reason to disregard the possibility of $d_{j}$ dominating the product, the other terms in the product themselves cannot together be non-negligible. ${ }^{15}$ However, it might be argued that there is a connection between $d_{j}$ and $y_{i}$ on the grounds that the probability of scientists not having enough resources to find alternatives is small. Of course this rejoinder ultimately depends on how the MIA is formalised and used to strengthen the connection between $d_{j}$ and $y_{i}$. Until that project is completed, a discussion of this argument is premature, hence beyond the scope of this paper. ${ }^{16}$

The more significant problem becomes clear when we look at the second component and then determine what it would take for (2) to have a sufficiently high value. Leaving aside the problem of evaluating $d_{j}$, it would have to be the case that at least one of the three factors, $y_{i} y_{k},\left(t_{i}-t_{k}\right)$ or $\left(f_{i j}-f_{k j}\right)$ dominates the product. For a fixed value of $D_{j}$, a high value of $\left(f_{i j}-f_{k j}\right)$ requires either an inequality of the form $i \ll k$ or that $f_{i j}$ takes a very specific form. Let us examine why this is the case. The condition that the function is non-increasing in $k$ is true for all values of $k$. This means that there cannot be a decrease in the value of $f_{i j}$ which is not followed by a further non-increase in the function. In order for the difference $\left(f_{i j}-f_{k j}\right)$ to have a non-zero value, it must, therefore, decrease in some interval between $i$ and $k$ (this condition is explicitly put in as a requirement in A5).

Let us now consider the possible forms of the function $y_{k}$. In order to aid our analysis, let us impose a further constraint on our functions - that they be convergent. ${ }^{17}$ In addition,

\footnotetext{
15 This amounts to granting the truth of P3.

${ }^{16}$ I am grateful to Richard Dawid for pointing this out to me.

17 Their domain is the natural numbers, so these functions are automatically continuous, because the preimage of any open set in the target is an open set in the domain equipped with the discrete topology.
} 
let them converge to zero for decreasing functions. ${ }^{18}$ For this function to take a suitably high value, it must be the case that there exist two values, $i$ and $k$ such that $y_{i}$ and $y_{k}$ are 'large'. This can be achieved by gerrymandering either our $y_{i}$ function or by choosing values of $i$ and $k$ in just the right way.

Let us consider the two sets of functions being modelled here. For the purpose of this argument, one only need consider either $t_{i}$ or $f_{i}$. Assume that $t_{i}$ has a significantly high value at some point where we are considering the value of the product. In such a case, confirmation will still be negligible if the product of $y_{i}$ and $f_{i}$ is negligible. And in the alternative case, where $t_{i}$ takes a negligibly low value at the relevant point, the product is doomed to give us negligible confirmation anyway. In other words, of the three functions, two pairs are such that the product of their functions cannot be significant. So it does not matter which of the pairs we consider.

Let us consider two extreme cases for the behaviour of $f_{i}$, and see how the $y_{i}$ functions respond, if they are modelled as monotonically increasing (other forms of $y_{i}$ are considered in the next section):

\section{Case 1-Linear monotonic functions}

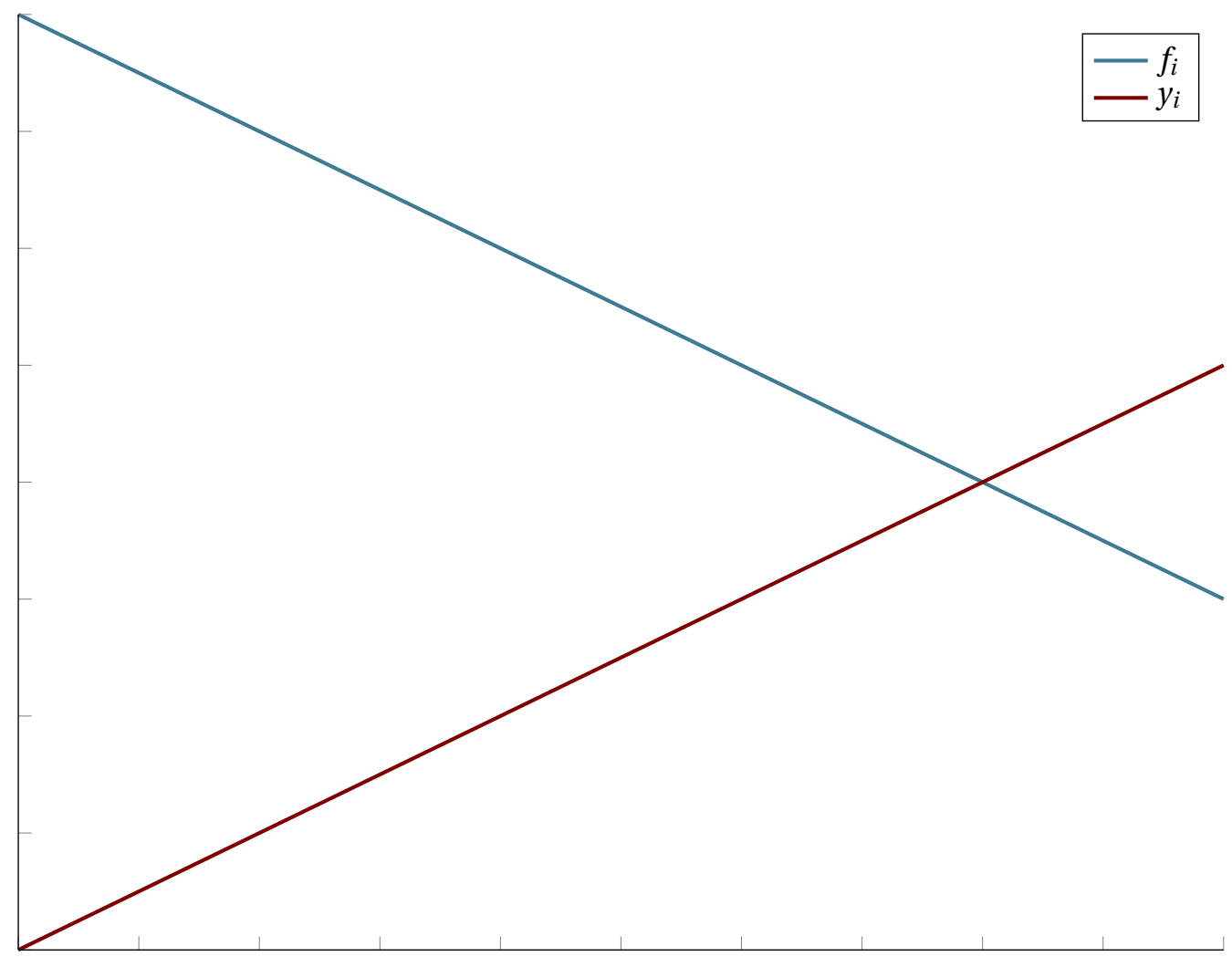

Consider representing both $f_{i}$ and $y_{i}$ as linear functions with a very mild gradient,

18 This condition has to be met for any function that does not assign a zero value to a finite collection of domain values in order for the Kolmogorov axioms to be satisfied. 
negative for $f_{i}$ and positive for $y_{i} \cdot{ }^{19}$ In such a case, if $i \ll k$, then $y_{i} y_{k}$ becomes negligibly small, for small values of $i$. For any nearby values of $i$ and $k$, the value of $f_{i}$ becomes negligible. Finally, for larger values of $i$, the condition that $y_{i}$ be a probability density function ensures that, beyond a certain finite value, the value of $y_{i}$ either drops to zero or approaches it asymptotically. This means that, for the proponent of the view that there are only finitely many alternatives, ${ }^{20}$ Dawid's analysis renders the confirmation negligible for any values of $i$ and $k$ beyond this limit.

\section{Case $2-f_{i}$ decays rapidly, $y_{i}$ increases monotonically}

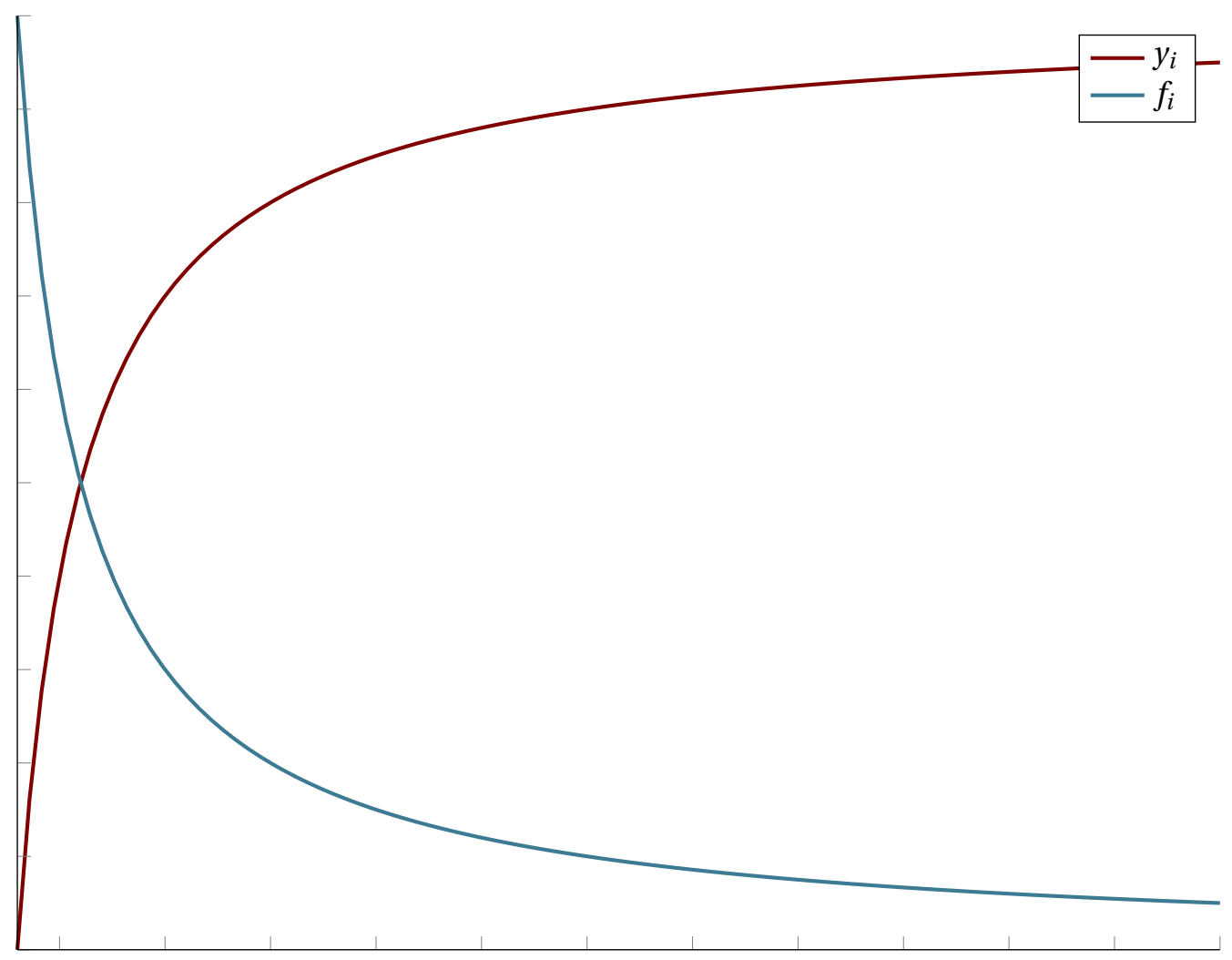

Here, in order that $\left(f_{i}-f_{k}\right)$ be large, we need it to be the case that either $i$ or $k$ is very low, and this suppresses $y_{i} y_{k}$. In the region where both $i$ and $k$ are low, there is still a chance that $f_{i}-f_{k}$ is significant, but this corresponds to a negligible value of $y_{i}$. If $i$ and $k$ are both very high, this suppresses $\left(f_{i}-f_{k}\right)$. And if $i \ll k$, then either $\left(f_{i}-f_{k}\right)$ is very low (if $i$ and $k$ are both high) or, as above, $y_{i} y_{k}$ is very low (if $i$ is very low). Once again, given the $y_{i}$ is a probability distribution, it cannot increase forever, so there will be some value of $i$ beyond which $y_{i} y_{k}$ will anyway be very small.

\footnotetext{
${ }^{19}$ In both of the following cases, note that, since $y_{i}$ is a probability density function, it will eventually, above a certain value, have to take on zero value, although it is allowed to do this asymptotically. This feature is not represented in the graphs.

${ }^{20}$ Bear in mind that Dawid's analysis does not work in the case that there are definitely infinitely many alternatives.[7, p. 216]
} 
There are, of course, variations on these function types available, but the two cases mrentioned above represent two extremes for the behaviour of the $\left(f_{i}-f_{k}\right)$ function. Given this restriction on the behaviour of that function, if $y_{i}$ is a monotonically increasing function (up to a certain value of $i$ ), confirmation is doomed to be negligible. The only way around is to assume that $y_{i}$ is not monotonic (or that it decays exponentially, which is a special case of case 3, described below.) But such functions fall prey to the gerrymandering objection.

\subsection{The Gerrymandering objection}

There are some values that the functions take that can plausibly be taken to lead to nonnegligible confirmation (the simplest example would be if $y_{i}=0, \forall i>0$ ). But the only way to do this is to impose very stringent restrictions on the form of the probability density functions in a way that begs the question against the whole project.

\section{Case $3-f_{i}$ decays very rapidly, while $y_{i}$ has a sudden spike for a small subset of values of $i$}

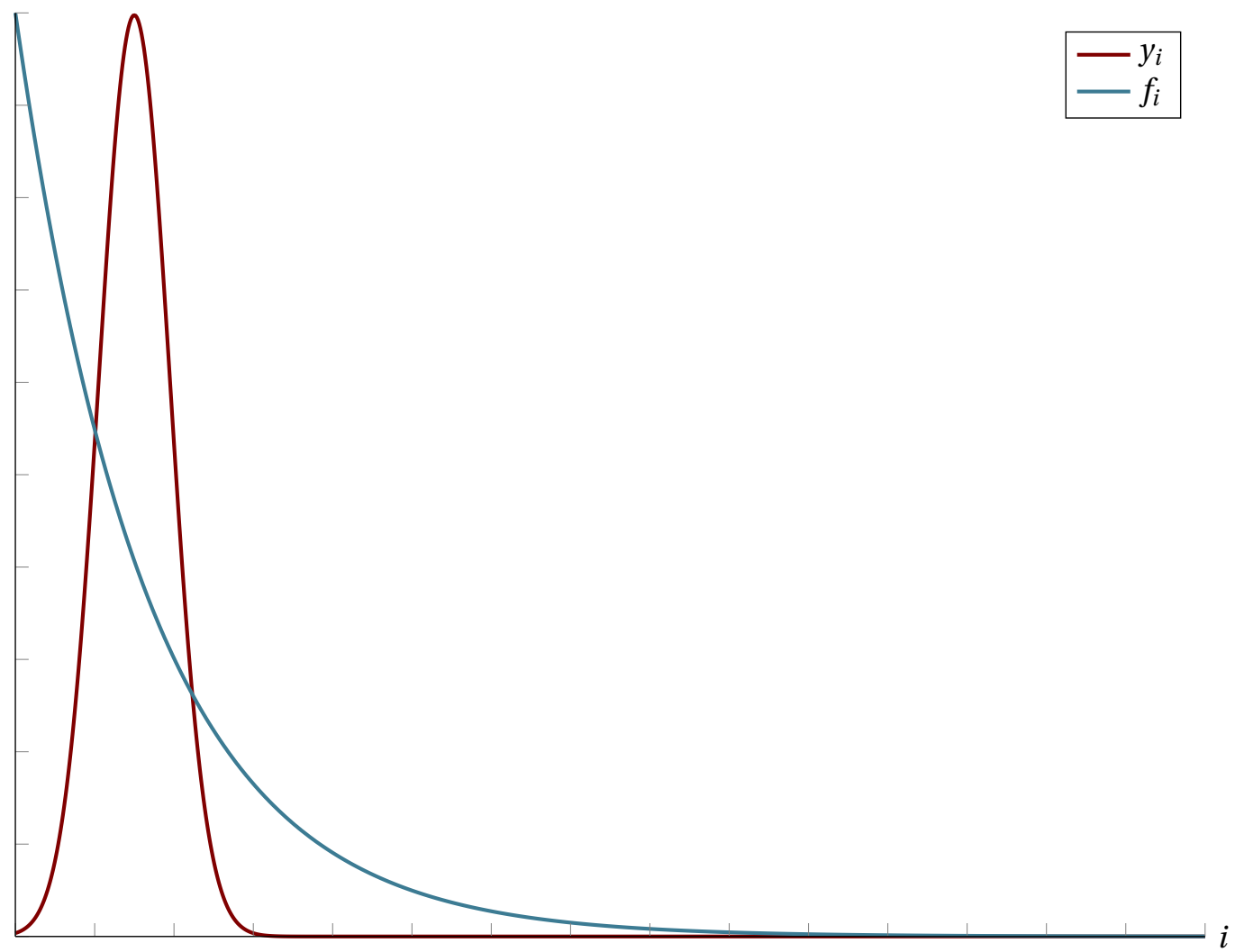

This is, formally, the most promising option so far. Here, for a pair of values $i, k$ that are low, and relatively near one another, two functions could take on values which, when multiplied, give us non-negligible confirmation. It is at this point, that we need to look at the plausibility of modelling our functions in this way. 
Consider first the $y_{i}$ function. Such a function models the proposition that the probability that there are no alternatives to the hypothesis $H$ is zero, but the probability that there are a few, say three or four, is very high. It goes on to suggest that the probability that there are more alternatives than three is low. In order for the value of the probability function to be sufficiently high at low values of $i$ and $k$, it needs to be the case that the probability function drops off rapidly after three or four (or whichever value of $i$ to which we attribute a spike). If not, then any value of $y_{i} y_{k}$ is doomed to be negligibly small. The problem with imposing this condition is that it begs the question of the whole project-whatever interval of the natural numbers corresponds to the spike in our function, it needs to be imposed pre-theoretically, specifically at a very low value of $i$. In other words, we cannot use Dawid's analysis to conclude that the No Alternatives Argument is valid, unless we have already assumed that there are no or few alteratives to $H$. This undermines the premise P2 of $\$ 2$.

\section{Case $4-f_{i}$ decays monotonically, while $y_{i}$ has two (or, more generally, $n$ sudden spikes for small subsets of values of $i$ and $k$ that are very far apart}

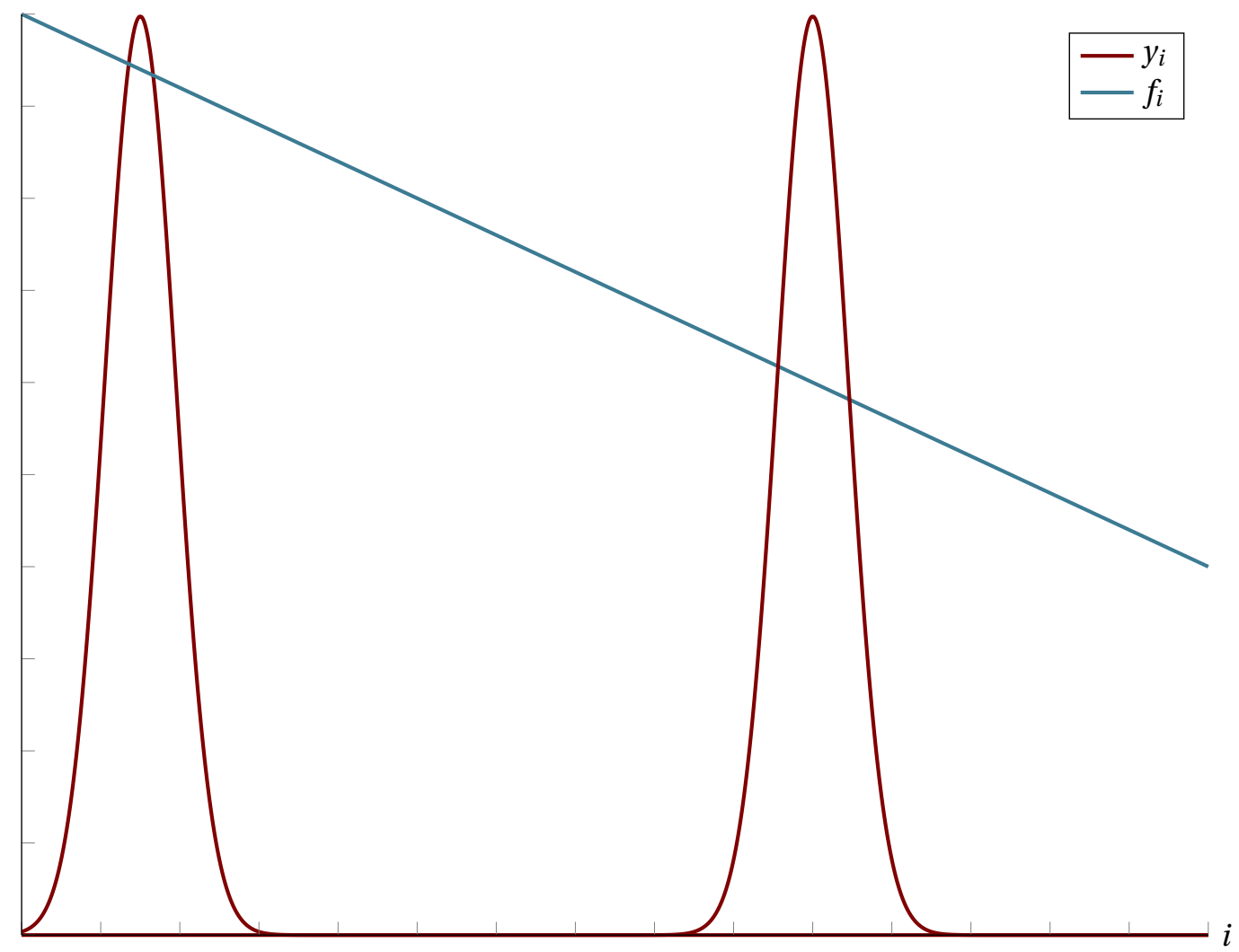

This case, a generalisation of case 3, lifts the restriction of (rapid) exponential decay of $f_{i}$ but imposes a highly unnatural constraint on $y_{i}$. In effect, we replace the no alternatives assumption with the 'either very few or some specific other number of alternatives' assumption. Modelling the $y_{i}$ function in this way is even more egregious than in case 3 , because there is no plausible justification for doing so coming from within the theory 
(or, for that matter pretheoretically). As in case 3, the second we impose unjustified extra constraints, we run the risk of begging the question, and undermining the purported soundness of the NAA.

Dawid's assertion that $P(T \mid F)-P(T)>0$ requires, for non-negligible confirmation, not only that $y_{i}$ be large, but that $f_{i j}$ and $t_{i j}$ be large too. This feature of the mathematics undermines P5 of the No Alternatives Argument by blocking the inference from 'there are few or no alternatives' to 'scientists should have a high degree of trust'. The former corresponds to $y_{i}$ taking on a high value for some small $i$, while the latter corresponds to $t_{i}$ and $f_{i}$, in addition to $y_{i}$ taking a high value for the same value of $i$. And the list of plausible cases presented in this paper demonstrate that this is not possible.

A second strand to the gerrymandering objection concerns the possibility that, although individual terms are negligible, their infinite sum might not be. Call this case $5 .^{21}$

For the sake of argument, let us assume that we have stipulated a sharp value, $k$, such that if $P(T \mid F)-P(T)>k$, then confirmation is non-negligible. $P(T \mid F)-P(T)$ is an infinite sum, so there are three ways in which it can take a value of $k$ or more:

(1) A single term in the summation is greater than or equal to $k$

(2) No single term is $\geq k$, but a finite sum of terms is greater than or equal to $k$. This requires a finite number of terms to be non-negligible.

(3) All individual terms are negligible, but the infinite sum is greater than or equal to $k$.

The first part of the gerrymandering objection explicitly argues against (1) and (2), in the retstricted context of the values of the functions at specific values of $i$.

Consider (3). Recall that $P(T \mid F)-P(T)$ is the difference between two infinite sums, $\Sigma\left(f_{i} \cdot y_{i} \cdot y_{k}\right)-\Sigma\left(f_{j} \cdot y_{i} \cdot y_{k}\right)$. In order for this to be non-negligible, a necessary (though, obviously not sufficient) condition is that the first sum be non-negligible. Rewrite the product function in the bracket of the first sum as, say $g(i)$, where $i$ is a positive integer. Only a specific number (which might, indeed, will be infinite) of functions $g(i)$ will sum non-negligibly. So non-negligible confirmation will still require a stipulation of a form that the $g(i)$ takes. And this, too, is subject to the gerrymandering objection, but now as applied to the form of the function, independent of the values it may take for a given integer.

The argument against a finite number of terms summing non-negligibly can be summarised more formally in the following way:

P1'. Cases 1, 2, 3, 4 exhaustively characterise the classes of plausible probability (density) functions.

\footnotetext{
${ }^{21} \mathrm{I}$ am grateful to $\mathrm{X}$ for drawing my attention to this possibility.
} 
P2'. Cases 1 and 2 cannot provide non-negligible confirmation (From the 'significance' part of the gerrymandering objection)

P3'. Confirmation can be non-negligible only if the functions are of the form presented in case 3 , case 4 .

P4'. Cases 3 and 4 apply only if one assumes that the $y_{i}$ takes a certain, very specific form. (From the gerrymandering objection)

P5'. For case 3, this form amounts to imposing the constraint that it is likely that there are no (or few) alternatives to $H$.

P6'. For case 4, this form amounts to imposing the constraint that the $y_{i}$ functions spike at intervals separated by a large distance.

P7'. Both P5' and P6' involve the assumption that it is likely that there are no alternatives, while $\mathbf{P 6}$ ' assumes, in addition, that it is also likely that there is a specific large number of alternatives.

Conclusion. Confirmation is non-negligible only if one assumes it is likely there are no (or few) alternatives.

Looking at premise P5', related to Case 3, one might argue that the assumption of no (or few) alternatives is a sufficient but not necessary assumption to constrain $y_{i}$ in the required way. Is it not enough, the objection goes, that the function have a spike somewhere, even if it is not the case that the precise location is pretheoretically determined? This is true, and, in the absence of constraints (or in the presence of different constraints) on $f_{i}$, this would, indeed, be enough. But the stringent restriction on $f_{i}$, that it be a non-increasing probability density function restricts the potentially useful $y_{i}$ functions to one of only two forms, both of which have to assume that it is likely that there are no or few alternatives to $H$.

In incorporating this assumption, we are forced to concede that the empirical basis for the claim P1 (that scientists have not found alternative hypotheses to $H$ despite considerable effort) is irrelevant to the argument which concludes that scientists should have a high degree of trust in $H$. The statement $Y_{k}$ of the form 'there are $k$ alternatives to $H$ ' is taken to be true, and no longer mediates the link between $H$ and $F$. As a result, the NAA is only a sound argument under the assumption that there are no or few alternatives, having lost the meta-empirical link from P1 to P2:

Q1. It is likely that there are few or no alternatives to $H$

Q2. If it is likely that there are few or no alternatives to $H$, then scientists should have a high degree of trust in $H$. 
Conclusion Scientists should have a high degree of trust in $H$.

In case 4 , in order for $y_{i} y_{k}$ to be non-negligible, $y_{i}$ will, in general, have to be nonnegligible (unless $y_{k}$ is very close to 1 , which would require some very strong pretheoretic arguments). If it turns out the case 4 accurately describes our situation, then that, on its own, undercuts the NAA, because the updated version of premise P2 now ought to read 'if scientists have not yet found an alternative to $H$, then it is likely either that there are few or no alternatives to $H$ or that there is some specific other number of alternatives to $H^{\prime}$. This means that $\mathbf{P 3}$ will have to be modified to read 'if it is likely that there are either few or no alternatives to $H$ or that there is a specific number of alternatives to $H$, then scientists should have a high degree of trust in $H$. At this point, this is no longer a sound argument in the way the the original NAA purported to be, given the implausibility of the updated P3.

Ultimately, what these cases go to show is that the requirement of non-negligibility, however it is framed, relies on the $f_{i j}$ function having a rapidly decaying form, in addition to which, the $y_{i}$ must peak at a low value of $i$. Importantly, no assumptions have to be made about the behaviour of these functions beyond those made by Dawid et al.-in this paper, I have not proposed a plausible model for either function, and used that to argue against Dawid. Instead, the significance component of the objection blocks any forms of $y_{i}$ that are not pretheoretically justified, while the gerrymandering component of the objection blocks the rest.

Before concluding, I would like to discuss a possible response to the analysis presented in this paper. ${ }^{22}$ Dawid et al's explicit intention behind formalising the NAA was to prove the possibility of non-empirical theory confirmation, but their paper can also be read as a demonstration of the possibility of non-negligible confirmation. In terms of the formal setup of this paper, their claim can be seen as an existence claim - there exist appropriate probability distributions that lead to significant confirmation. And there is nothing that rules out, a priori, the above examples from as corresponding the appropriate priors. So, one might argue, a hypothesis can, in principle, receive non-negligible confirmation from the realisation that there are no viable alternatives.

As it stands, this reasoning is valid. Indeed, this is just a rephrasing of P5. However, it is useful to consider why this is not a good response to the analysis in this paper by looking at the broader context of the project. Our interest in confirmation stems from, among other things, a desire to justify our acceptance of a particular theory. Our claims to having scientific knowledge are traditionally made by reference to some experimental observation that justifies our acceptance of the scientific theory on the grounds that that theory predicts that observation.

Limitation to scientific underdetermination plays a different role to experimental observation, since no theory predicts the extent to which it limits scientific underdetermination. Logically, the structures are as follows. For experimental confirmation,

\footnotetext{
${ }^{22}$ Thanks to an anonymous reviewer for raising this point.
} 
(i) $H \rightarrow E$, where $H$ is a hypothesis and $E$ is some empirical observation,

whereas for non-empircal confirmation

(ii) $N A A \rightarrow H$, where $N A A$ is the claim that there are no alternatives to $H$.

In (i), justification of belief in the empirical adequacy of $H$ comes from accumulation of evidence, $E$, combined with standard Bayesian confirmation analysis. In the case of (ii), belief in $H$ is supported, but only to the extent that we have arguments supporting the antecedent ((ii) is the same as P5). Merely stipulating that the antecedent is true will, by modus ponens, mean that the consequent is also true. But the justification of this claim is now suspect, because we are, in effect, just stipulating that $H$ is true. It might turn out that this is correct; $H$ might, by some stroke of luck, happen to be the empirically adequate theory we seek. But in this case, the NAA no longer plays the role of justifying our acceptance of $H$, rather, it provides a grandiose framework for stating the simple stipulation that we had already accepted $H$ to begin with.

\section{Conclusion}

I have focussed my scepticism about Dawid's programme of non-empirical theory confirmation on the qualitative Bayesian methodology used to argue in favour of non-empirical confirmation. More precisely, having made a distinction between negligible confirmation and non-negligible confirmation, I questioned the assumption that the NAA could be the lynch pin of a non-negligible confirmation programme. The soundness of the NAA requires that the link between a theory being confirmed and the trust that scientists have in the theory be robust. It ceases to be sound, either because (i) the significance objection blocks P3: 'If it is likely that there are few or no alternatives to $H$, then scientists should have a high degree of trust in $H^{\prime}$ ' or (ii) because the gerrymandering objection means that the consequent of $\mathbf{P 2}$ is merely asserted: 'If scientists have not yet found an alternative to $H$, then it is likely that there are few or no alternatives to $H$.'

Without the NAA to serve as its cornerstone, Dawid's programme of non-empirical theory confirmation must rely on some quantitative, non-negligible confirmation from a formalisation of one of its other two pillars, the UEA or the MIA. Future work will determine whether the programmes of formalising those two arguments are successful; either way the result of this paper is intended to show that formalisation of the NAA, at least in the form presented by Dawid et al. will not feature in a successful formalisation of Dawid's overall programme.

\section{Acknowledgements}




\section{References}

[1] Richard Dawid. Underdetermination and theory succession from the perspective of string theory. Philosophy of Science, 73(3):298-322, 2006.

[2] Richard Dawid. Scientific realism in the age of string theory. Physics and Philosophy, 11:1-32, 2007.

[3] Richard Dawid. String theory and the scientific method. Cambridge University Press, 2013.

[4] Richard Dawid. Theory assessment and final theory claim in string theory. Foundations of Physics, 43(1):81-100, 2013.

[5] Richard Dawid. Modelling non-empirical confirmation. In Ippoliti et al, editor, Models and Inferences in Science. Springer, New York, NY, 2016.

[6] Richard Dawid. The Significance of Non-Empirical Confirmation in Fundamental Physics. Preprint available at http://philsci-archive.pitt.edu/12791/, February 2017.

[7] Richard Dawid, Stephan Hartmann, and Jan Sprenger. The no alternatives argument. The British Journal for the Philosophy of Science, 66(1):1-22, 2014.

[8] Benjamin Eva and Stephan Hartmann. When no reason for is a reason against. Analysis, 78(3):426-431, 2018.

[9] Carl G Hempel. Studies in the logic of confirmation (i.). Mind, 54(213):1-26, 1945.

[10] Tushar Menon. A reason against 'when no reason for is a reason against'. MS, 2018.

[11] Philipe van Basshuysen. Dawid et al.'s [2015] no alternatives argument: an empiricist note. Kriterion: Journal of Philosophy, 29(1):37-50, 2015.

[12] Bas C Van Fraassen. The scientific image. Oxford University Press, Oxford, 1980.

[13] Peter Vickers. Understanding inconsistent science. Oxford University Press, Oxford, 2013. 